\title{
EXISTENCE AND UNIQUENESS OF FLOWS BEHIND THREE-DIMENSIONAL STATIONARY AND PSEUDO-STATIONARY SHOCKS ${ }^{1}$
}

\author{
R. P. KANWAL
}

1. Introduction. Recently we have discussed the flows immediately behind three-dimensional shock waves in steady $[1 ; 2]$ as well as pseudo-stationary $[3]$ gas flows. In that analysis certain shock surfaces were found behind which the flow remains irrotational. The purpose of this paper is to show that the integration of the various conservation equations is equivalent to the solution of a Cauchy problem with the shock front as surface on which the initial data is given. By Cauchy-Kowaleski theorem it is proved that in a neighborhood of the shock the flow behind the shock exists and is uniquely determined. The corresponding case for two-dimensional gas flows has been analysed by Taub [4].

The paper has been divided into two parts. First part deals with the stationary flows, while the second part deals with the pseudostationary flows. The detailed analysis is given only for the stationary flows. Formal methods of tensor analysis have been used throughout the paper.

\section{Part I. Stationary flows}

2. Equations of motion and shock conditions. We consider the equations governing the flow of a stationary ideal gas with viscosity and thermal-conductivity zero, namely

$$
\begin{aligned}
\rho_{, i} u_{i}+\rho u_{j, j} & =0, \text { (equation of continuity) } \\
p_{, i}+\rho u_{j} u_{i, j} & =0, \text { (equations of motion), } \\
S_{, i} u_{i} & =0, \text { (changes of state are adiabatic), }
\end{aligned}
$$

in which $p, \rho, u_{i}$ and $S$ denote the pressure, density, velocity components and entropy respectively. We assume the motion referred to a system of rectangular coordinates $x^{i}$.

If a shock wave arises, it will be assumed that this divides the flow under consideration into a region 1 and a region 2 . We suppose the region 1 to be traversed by the gas before contact with the shock wave

Received by the editors September 3, 1957 and, in revised form October 2, 1957.

1 This research was carried out under U. S. Army Contract No. DA-11-022-ORD2059 with the University of Wisconsin. 
and region 2 to be that in which the gas enters after passing through it. Denote by $u_{1 i}, \rho_{1}$ and $p_{1}$ the velocity components, density, and pressure on the wave bordering region 1 and correspondingly by $u_{2 i}$, $\rho_{2}$ and $p_{2}$ the values of these quantities on the side of the wave bordering the region 2. Put

$$
u_{1 n}=u_{1 i} n_{i} ; \quad u_{2 n}=u_{2 i} n_{i},
$$

where $n_{i}$ are the components of the unit normal to the shock surface, directed from region 1 into region 2. Then the Rankine-Hugoniot shock conditions for the stationary wave under consideration can be written in the form

$$
\left[u_{i}\right]=\frac{-\delta u_{1 n} n_{i}}{1+\delta}, \quad[p]=\frac{\delta \rho_{1} u_{1 n}^{2}}{1+\delta}, \quad[\rho]=\delta \rho_{1},
$$

where

$$
\delta=\frac{2\left(\rho_{1} u_{1 n}^{2}-\gamma p_{1}\right)}{2 \gamma p_{1}+(\gamma-1) \rho_{1} u_{1 n}^{2}},
$$

and the bracket [ ] denotes the difference of the values on the two sides of the shock surface of the quantity enclosed. In the subsequent discussion, for simplicity, we shall omit the subscript 2 on the quantities appearing in the left members of these equations.

3. The new coordinate system. The shock configuration in a threedimensional flow may be represented by the equations [3]:

$$
x_{i}=a_{i}\left(y_{1}, y_{2}\right), \quad i=1,2,3,
$$

where $y_{\alpha}(\alpha=1,2)$ are the Gaussian coordinates on the shock surface.

Extending Taub's analysis [4], we define a transformation $x_{i}$ $=a_{i}\left(y_{1}, y_{2}, T\right)$, from the variables $x_{i}$ to a new set of variables $y_{\alpha}$ and $T . T$ denotes the arc length of the streamlines behind the shock surface and $a_{i}$ are the solutions of the equations

$$
\frac{\partial a_{i}}{\partial T}=\frac{u_{i}}{v}, \quad v^{2}=u_{i} u_{i}
$$

with the initial condition

$$
a_{i}\left(y_{1}, y_{2}, 0\right)=a_{i}\left(y_{1}, y_{2}\right) .
$$

The functions on the right hand side of this equation are the functions entering equations (6). Let us further define 


$$
\begin{aligned}
\partial a_{i} / \partial y^{\alpha} & =A_{i \alpha}, \\
A_{i \alpha}\left(y_{1}, y_{2}, 0\right) & =\alpha_{i \alpha},
\end{aligned}
$$

where $a_{i \alpha} a_{i \beta}=g_{\alpha \beta}$ are the components of the first fundamental form of the shock surface [5].

From (7) and (9) we have

$$
\frac{\partial A_{i \alpha}}{\partial T}=\frac{\partial}{\partial y^{\alpha}}\left(\frac{u_{i}}{v}\right)
$$

Also

$$
A_{i \alpha, \beta}=B_{\alpha \beta} N_{i} / N
$$

where

$$
\begin{aligned}
N_{i} & =\frac{1}{2} \epsilon^{\alpha \beta} e_{i j k} A_{j \alpha} A_{k \beta} ; & & N^{2}=N_{i} N_{i}, \\
N_{i}\left(y_{1}, y_{2}, 0\right) & =n_{i}\left(y_{1}, y_{2}\right) ; & & A_{i \alpha} N_{i}=0,
\end{aligned}
$$

and $B_{\alpha \beta}$ are the components of the second fundamental form of the surface and $\epsilon^{\alpha \beta}$ and $e_{i j k}$ are the components of the surface and space permutation tensors, respectively (5).

In view of the above mentioned transformation we may write for any function

$$
f_{, i}=\frac{\partial f}{\partial T} T_{, i}+\frac{\partial f}{\partial y^{\alpha}} y_{, i}^{\alpha} .
$$

Putting $f=a_{i}$ and substituting from (7) and (9) we have

$$
\delta_{i j}=\frac{u_{i}}{v} T_{, j}+A_{i \alpha} y_{, j}^{\alpha} .
$$

Multiplying both sides of (15) by $N_{i}$ and $2^{-1} \epsilon^{\alpha \beta} e_{i j k} u_{l} A_{k \beta}$ in turn, making use of the geometrical relations (13) and the skew-symmetry of the permutations tensors, we get

$$
T_{, j}=\frac{v}{u_{n}} N_{j},
$$

and

$$
y_{, j}^{\alpha}=\frac{1}{2 u_{n}} \epsilon^{\alpha \beta} e_{i j k} A_{k \beta} u_{i}
$$

where

$$
u_{n}=u_{i} N_{i} .
$$


Thus

$$
\begin{aligned}
u_{i, j} & =\frac{v N_{j}}{u_{n}} \frac{\partial u_{i}}{\partial T}+y_{, j}^{\alpha} \frac{\partial u_{i}}{\partial y^{\alpha}} \\
u_{i, j} u_{j} & =v \frac{\partial u^{i}}{\partial T} .
\end{aligned}
$$

Furthermore it follows from equations (16) and (17) that for an arbitrary function $f$ we have

$$
u_{i} f_{, i}=v \frac{\partial f}{\partial T}
$$

Now with the help of the equations (19) and (21) the equations (1) to (3) are transformed respectively to

$$
\begin{gathered}
\frac{\partial}{\partial T}\left(\rho u_{n}\right)=0 \\
\frac{\partial u_{i}}{\partial T}+\frac{N_{i}}{\rho u_{n}} \frac{\partial p}{\partial T}=\frac{1}{\rho v} \frac{\partial p}{\partial y^{\alpha}} y_{, i}^{\alpha}
\end{gathered}
$$

and

$$
v \frac{\partial}{\partial T}\left(p \rho^{-\gamma}\right)=0
$$

Noting that $u_{n}=2^{-1} \epsilon^{\alpha \beta} e_{i j k} u_{i} A_{j \alpha} A_{k \beta}$, and making use of the relation (11) and the skew-symmetry of the quantities $\epsilon^{\alpha \beta}$ and $e_{i j k}$, the equation (22) reduces to

$$
\frac{u_{n}}{\rho} \frac{\partial \rho}{\partial T}+N_{i} \frac{\partial u_{i}}{\partial T}=-\frac{\epsilon^{\alpha \beta} e_{i j k} u_{i} A_{k \beta}}{v} \frac{\partial u_{j}}{\partial y^{\alpha}}
$$

Furthermore equation (24) gives

$$
p=p_{0}\left(\rho / \rho_{0}\right)^{\gamma}
$$

where $p_{0}$ and $\rho_{0}$ are the values of the pressure and density at $T=0$, i.e., on the shock surface and are functions of $y_{\alpha}$.

If we put $\xi=\log (p) / \gamma$ then it follows from (26) that

$$
\frac{1}{\rho} \frac{\partial \rho}{\partial T}=\frac{1}{\gamma p} \frac{\partial p}{\partial T}=\frac{\partial \xi}{\partial T} .
$$

With the help of the relations (27) and (19), the equations (25) and (23) can respectively be written as 


$$
u_{n} \frac{\partial \xi}{\partial T}+N_{i} \frac{\partial u_{i}}{\partial T}=-\frac{\epsilon^{\alpha \beta} e_{i j k} u_{i} A_{k \beta}}{v} \frac{\partial u_{j}}{\partial y^{\alpha}}
$$

and

$$
c^{2} N_{i} \frac{\partial \xi}{\partial T}+u_{n} \frac{\partial u_{i}}{\partial T}=-\frac{c^{2} \epsilon^{\alpha \beta} e_{i j k} u_{i} A_{k \beta}}{2 v} \frac{\partial \xi}{\partial y^{\alpha}},
$$

where $c^{2}=\gamma p / \rho$.

Now if an analytic shock surface is given and if flow incident upon it is also given, i.e., if we are given $a_{i \alpha}, u_{1 i}, p_{1}$ and $\rho_{1}$, then the CauchyKowaleski existence theorem for equations (28) and (29) enables us to state that there exists a unique analytic flow in a region behind the shock front provided that

$$
v^{4} \operatorname{det}\left\|\begin{array}{cccc}
u_{n} & N_{1} & N_{2} & N_{3} \\
c^{2} N_{1} & u_{n} & 0 & 0 \\
c^{2} N_{2} & 0 & u_{n} & 0 \\
c^{2} N_{3} & 0 & 0 & u_{n}
\end{array}\right\|=v u_{n}^{4}\left(1-\frac{c^{2} N^{2}}{u_{n}^{2}}\right) \neq 0
$$

i.e.,

$$
1-\frac{c^{2} N^{2}}{u_{n}^{2}} \neq 0
$$

If we make use of the shock relations (4) and the fact that $N^{2}=n^{2}=1$, at the shock front, we readily obtain

$$
1-\frac{c^{2} N^{2}}{u_{n}^{2}}=-\frac{\gamma+1}{2} \delta .
$$

Since $\delta>0$ for a shock of nonzero strength, the required condition is fulfilled. Thus there exists a unique analytic flow in a region behind a steady shock wave.

\section{Part II. Pseudo-stationary flows}

In this case the three-dimensional shock configuration at time $t$ may be represented by the equations [3]:

$$
x_{i}=t a_{i}\left(y_{1}, y_{2}\right)
$$

where $y_{1}, y_{2}$ are the Gaussian coordinates on the shock surface. The equations describing the motion of a fluid for a pseudo-stationary flow are then given as $[3 ; 6]$ : 
(34) $\rho U_{i, i}+\rho_{, i} U_{i}+3 \rho=0$, (equation of continuity),

(35) $\rho\left(U_{i, j} U_{j}+U_{i}\right)+p_{, i}=0$, (equations of motion),

$$
S_{, i} U_{i}=0 \text {, (the changes of state are adiabatic), }
$$

where $U_{i}=u_{i}-a_{i}$. The shock conditions (4) remain the same except that $u_{i}$ is changed to $U_{i}$ in them [3]. In these equations comma denotes differentiation with respect to $a_{i}$.

If $T$ represents the arc length along the streak line, a line having $U_{i} / v$ as the components of its unit tangent vector, measured from the shock front [4], then we define, as in Part I, the transformation from $a_{i}$ to $y_{1}, y_{2}$ and $T$ such that $a_{i}$ are the solutions of the equations

$$
\frac{\partial a_{i}}{\partial T}=\frac{U_{i}}{v} ; \quad v^{2}=U_{i} U_{i},
$$

with the initial condition: $a_{i}\left(y_{1}, y_{2}, 0\right)=a_{i}\left(y_{1}, y_{2}\right)$.

The transformation from the variables $a_{i}$ to the variables $y_{1}, y_{2}, T$ thus completely defined, we proceed in a manner exactly similar to the one in Part I. The conservation equations (34) to (36) are transformed respectively into

$$
\begin{aligned}
\frac{U_{n}}{\rho} \frac{\partial \rho}{\partial T}+N_{i} \frac{\partial U_{i}}{\partial T}+\frac{3}{v} & =-\frac{\epsilon^{\alpha \beta} e_{i j k} U_{i} A_{k \beta}}{v} \frac{\partial U_{j}}{\partial y^{\alpha}}, \\
\frac{\partial U_{i}}{\partial T}+\frac{U_{i}}{v}+\frac{N_{i}}{\rho U_{n}} \frac{\partial p}{\partial T} & =-\frac{\epsilon^{\alpha \beta} e_{i j k} U_{j} A_{k \beta}}{2 \rho v} \frac{\partial p}{\partial y^{\alpha}}, \\
p & =p_{0}\left(\rho / \rho_{0}\right)^{\gamma} .
\end{aligned}
$$

As in the stationary case if we put $\xi=\gamma^{-1} \log p$ and $c^{2}=\gamma p / \rho$ then these equations reduce to

$$
U_{n} \frac{\partial \xi}{\partial T}+N_{i} \frac{\partial U_{i}}{\partial T}=-\frac{\epsilon^{\alpha \beta} e_{i j k} U_{i} A_{k \beta}}{v} \frac{\partial U_{j}}{\partial y^{\alpha}}-\frac{3}{v},
$$

and

$$
c^{2} N_{i} \frac{\partial \xi}{\partial T}+U_{n} \frac{\partial U_{i}}{\partial T}=-\frac{c^{2} \epsilon^{\alpha \beta} e_{i j k} U_{j} A_{k \beta}}{v} \frac{\partial \xi}{\partial y^{\alpha}}-\frac{U_{i} U_{n}}{v} .
$$

Comparing the set of equations (41) and (42) with the set (28) and (29) we see that the condition for the applicability of the CauchyKowaleski existence theorem is the same in this case as in the case of stationary gas flows and is, therefore, satisfied. Hence, there exists a unique analytic flow behind a pseudo-stationary shock. 


\section{REFERENCES}

1. R. P. Kanwal, Shock and wave surfaces for three-dimensional gas flows, Ph.D. Thesis, Indiana University, 1957.

2. - On curved shock waves in three-dimensional gas flows, Presented at the American Mathematical Society meeting in April, 1957, at Chicago, to appear in the Quarterly of Applied Mathematics.

3. - Propagation of curved shocks in pseudo-stationary three-dimensional gas flows, to appear in Illinois Journal of Mathematics.

4. A. H. Taub, Determination of flows behind stationary and pseudostationary shocks, Ann. of Math. vol. 62 (1955) pp. 300-325.

5. L. P. Eisenhart, Introduction to differential geometry, Princeton University Press, 1941, Chapters III and IV.

6. A. H. Fletcher, A. H. Taub and W. Bleakney, The Mach reflection of shock waves at nearly glancing incidence, Reviews of Modern Physics vol. 23 (1951) pp. 271-286.

Mathematics Research Center, University of Wisconsin

\section{NOTE ON A PAPER OF CIVIN AND YOOD}

\section{LEONARD E. BAUM}

It is possible to obtain stronger results than those of [1] in a small amount of space through the use of a different method. We follow the notation of [1] throughout.

Theorem 1. Let B be a complex commutative Banach algebra with countable space TI of maximal regular ideals. Let $A$ be a separating subalgebra. Then (i) if $A$ is not contained in any maximal regular ideal it is determining. Otherwise (ii) there exists a (unique) maximal regular ideal $M$ of $B$ with $\hat{A}$ dense in $\hat{M}$.

Proof. If $x \in A$ then $\mathrm{Cl}[\hat{x}(\mathfrak{T})]=\hat{x}(\mathfrak{T}) \cup\{0\}$ is a countable compact set. Then by a theorem of Lavrent'ev [2], polynomials in $z$ are uniformly dense on $\mathrm{Cl}[\hat{x}(\mathfrak{T})]$ in all continuous complex valued functions on $\mathrm{Cl}[\hat{x}(\mathfrak{T})]$ and in particular uniformly approximate $\bar{z}$ on $\mathrm{Cl}[\hat{x}(\mathfrak{T})]$, i.e. polynomials in $\hat{x}$ uniformly approximate $\hat{x}^{-}$on $\mathfrak{T}$. Then, $\mathrm{Cl}[\hat{A}]$ being a uniformly closed, separating, self adjoint algebra of continuous, complex valued functions vanishing at $\infty$ on a locally compact space $\mathscr{T}$, equals $\mathfrak{C}(\mathfrak{T})$ in case (i) or at worst some unique $\mathrm{Cl}[\widehat{\mathfrak{N}}]$ in case (ii) by the Stone-Weierstrass approximation theorem [3].

Theorem 3.3 and extensions of Theorem 3.5 and Corollary 3.6 of [1] eliminating the hypothesis of regularity and the necessity of limiting the discussion to maximal subalgebras follow as simple corollaries, e.g.

Received by the editors January 29, 1957 and, in revised form, June 15, 1957. 